\title{
Contraindications to lumbar puncture as defined by computed cranial tomography
}

\author{
DJ GOWER, * AL BAKER, $\dagger$ WO BELL, ${ }^{*}$ MR BALL $\dagger$ \\ From the Section on Neurosurgery, Departments of Surgery,* and Radiology, $\dagger$ Wake Forest University Medical \\ Center, Bowman Gray School of Medicine, Winston-Salem, North Carolina, U.S.A.
}

SUMMARY Papilloedema is not always an adequate predictor of potential complications from lumbar puncture, and many clinicians are using computed tomography (CT) before lumbar puncture in an effort to identify more accurately the "at risk" patient. This paper identifies the following anatomical criteria defined by CT scanning that correlate with unequal pressures between intracranial compartments and predispose a patient to herniation following decompression of the spinal compartment: lateral shift of midline structures, loss of the suprachiasmatic and basilar cisterns, obliteration of the fourth ventricle, or obliteration of the superior cerebellar and quadrigeminal plate cisterns with sparing of the ambient cisterns. These criteria should be considered to be contraindications to lumbar puncture.

Lumbar puncture was originally described by Quincke in 1891 , and it has since proved to be a highly effective diagnostic procedure. ${ }^{1}$ In 1909 , Harvey Cushing recognized that patients with unequal pressures between intracranial compartments who underwent lumbar puncture were at risk for cerebral herniation and death. ${ }^{2}$ This group of patients was best identified at that time by the presence of a "choked disk" (papilloedema), and his finding resulted in the axiom that papilloedema was a contraindication to lumbar puncture.

More recently, some authors have suggested that papilloedema is not always an adequate predictor of complications from lumbar puncture, ${ }^{3}$ and many clinicians now turn to computed tomography (CT) before performing lumbar puncture. Most would agree that a "normal" CT scan indicates a patient at minimal risk for herniation, but a scan that shows some type of abnormalities, such as nonobstructive hydrocephalus, atrophy, or meningeal enhancement, may not clearly answer the question, "Is this patient safe to tap?".

The purpose of this paper is twofold: first, to report

\begin{abstract}
Address for reprint requests: Dr DJ Gower, Section on Neurosurgery, Bowman Gray School of Medicine, 300 South Hawthorne Road, Winston-Salem, NC 27103, USA.
\end{abstract}

Received 12 August 1986 and in revised form 24 November 1956. Accepted 28 November 1986 a case of death following lumbar puncture with premorbid CT definition of the intracranial anatomy and second, to suggest to the clinician criteria that may help to identify by CT those patients who are indeed at increased risk for complications from lumbar puncture.

\section{Case Report}

A 29 year old man developed a severe headache with associated nausea and vomiting on one day, and was lethargic on the next day. When examined in a local emergency room, he had a stiff neck, temperature of $40^{\circ} \mathrm{C}$, peripheral white blood cell count of 28,000 per $\mathrm{mm}^{3}$, and mild left hemiparesis. A diagnostic lumbar puncture was performed for suspected meningitis. The cerebrospinal fluid had 80 white blood cells per $\mathrm{mm}^{3}$ (16\% polymorphonuclear cells), glucose of $97 \mathrm{mg} / \mathrm{dl}$, and protein of $118 \mathrm{mg} / \mathrm{dl}$. The patient was being transported to the $x$-ray suite for an admission chest radiograph when he became unresponsive and developed fixed and dilated pupils with decerebrate posturing and respiratory compromise. An emergency CT scan was done and demonstrated a right thalamic mass lesion with shift of the midline structures (fig 1). He was transferred to our institution where he was treated aggressively for increased intracranial pressure with hyperventilation and mannitol, and for suspected purulent cerebritis with broad-spectrum antibiotics. He was declared brain dead 24 hours after admission, and life support systems were withdrawn at the family's request. Postmortem examination of the brain demonstrated a non-typed fungal cerebritis in the right thalamus. No source of fungal emboli was found. 


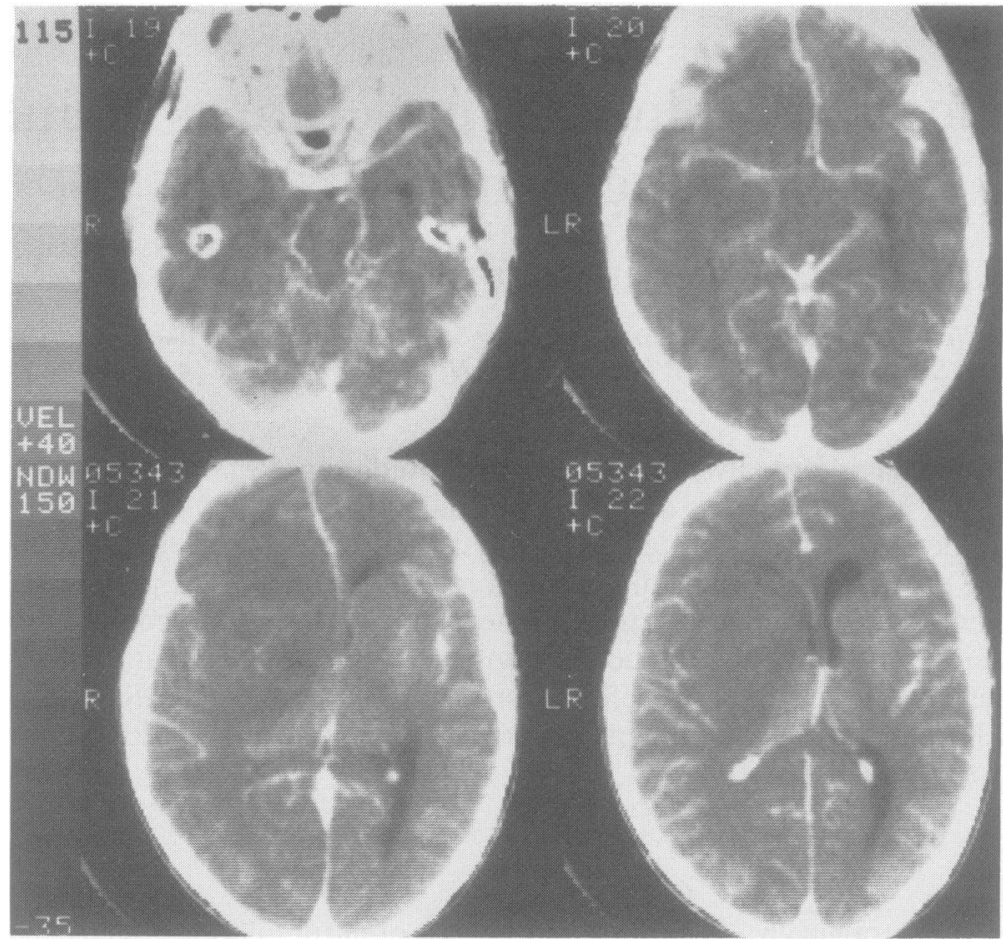

Fig 1 Infused CT scan that demonstrates midline shift and uncal herniation with displacement of the right posterior cerebral artery.

\section{CT evidence of unequal intracranial pressures}

Shifts of brain parenchyma between intracranial compartments in a patient with unequal intracranial pressures may compress neural structures against bony or dural prominences. ${ }^{2}$ Two common forms of neural compression occur: one when the medial portion of the temporal lobe herniates into the tentorial notch and compresses the midbrain, peduncle, and third nerve (uncal herniation); the other at the foramen magnum when the cerebellar tonsils compress the medulla (tonsillar herniation). Fluid shifts and pressure changes between compartments may occur whenever lumbar puncture is performed. The lowering of the spinal compartment pressure by the tap and removal of cerebrospinal fluid will cause some downward movement of the transtentorial or tonsillar structures, and for this reason all patients undergoing lumbar punctures are, theoretically, at some risk of cerebral herniation. Those predisposed by having unequal intracranial pressures and shift of cerebral tissue into close proximity to a compartment opening (tentorial hiatus, foramen magnum) are at a much greater risk.

Over the past 25 years, modifications of the tech- nique of spinal puncture have significantly reduced the risk for patients with normal intracranial anatomy and pressure. The "standard" needle is now 20 gauge, instead of the 12- or 16-gauge needle that was once used. ${ }^{3}$ Lafforgue and colleagues have shown that a smaller dural puncture site allows less cerebrospinal fluid to extravasate into the surrounding tissues following removal of the needle ${ }^{4}$ and current techniques for complete analysis of cerebrospinal fluid now require only 3 to $6 \mathrm{ml}$ of fluid instead of the 30 to $60 \mathrm{ml}$ routinely removed when the procedure was first used.

Despite these modifications, patients with unequal intracranial pressures are still at risk for cerebral herniation regardless of needle size; moreover, identification of these patients remains difficult. Korein et al, in a review of their own patients and patients reported in the literature between 1927 and $1959,{ }^{3}$ identified a total of 1053 who had undergone lumbar puncture. Four hundred and eighteen had papilloedema, of whom only five $(1 \cdot 2 \%)$ had a "possible complication" within the first 48 hours following lumbar puncture. This study confirmed a growing suspicion that the presence of papilloedema alone 
(although in some cases an indicator of increased intracranial pressure) may not be an adequate predictor of cerebral herniation following lumbar puncture.

While CT scanning frequently eliminates the need for diagnostic lumbar puncture by providing a firm diagnosis, such as subarachnoid haemorrhage or brain tumour, ${ }^{5}$ two clear indications for lumbar puncture remain: suspected purulent or carcinomatous meningitis, and suspected spinal cord compression from metastatic disease requiring myelography. For patients in both of these categories, a mass lesion from a brain abscess or an intraparenchymal metastatic lesion may make lumbar puncture treacherous.

Although the CT scan clearly defines intracranial anatomy, pressure data must be inferred from the structural information it provides. Thus, some authors have recommended that lumbar puncture be attempted only if the CT scan is "normal". ${ }^{6} \mathrm{We}$ agree that all patients should have both uninfused and infused CT scans before lumbar puncture, but limiting lumbar puncture to those with "normal" scans may not be necessary. We suggest the following CT criteria that are anatomical correlates to unequal intracranial pressures and should be considered contraindications to a proposed diagnostic lumbar tap.

\section{(1) CT evidence of unequal pressures across the midline} (falx cerebri)

The presence of unequal supratentorial pressures is easily identified by lateral shift of the midline structures (septum pellucidum, third ventricle). ${ }^{7}$ Asymmetry of the lateral ventricles alone may not be an accurate sign, since ipsilateral ventricular dilatation may occur secondary to stroke, or coaptation of a frontal horn may represent a normal anatomical variant. Following lumbar puncture unequal supratentorial pressures would most likely lead to compression of the ipsilateral temporal lobe, leading to an uncal herniation.

(2) CT evidence of unequal pressures between the supratentorial and infratentorial compartments

Unequal pressures across the tentorium may be subdivided into an elevated pressure cephalad to the tentorium pushing structures into the posterior fossa or an elevated pressure caudad to the tentorium pushing structures upward as well as through the foramen magnum.

Symmetrical supratentorial pressures higher than

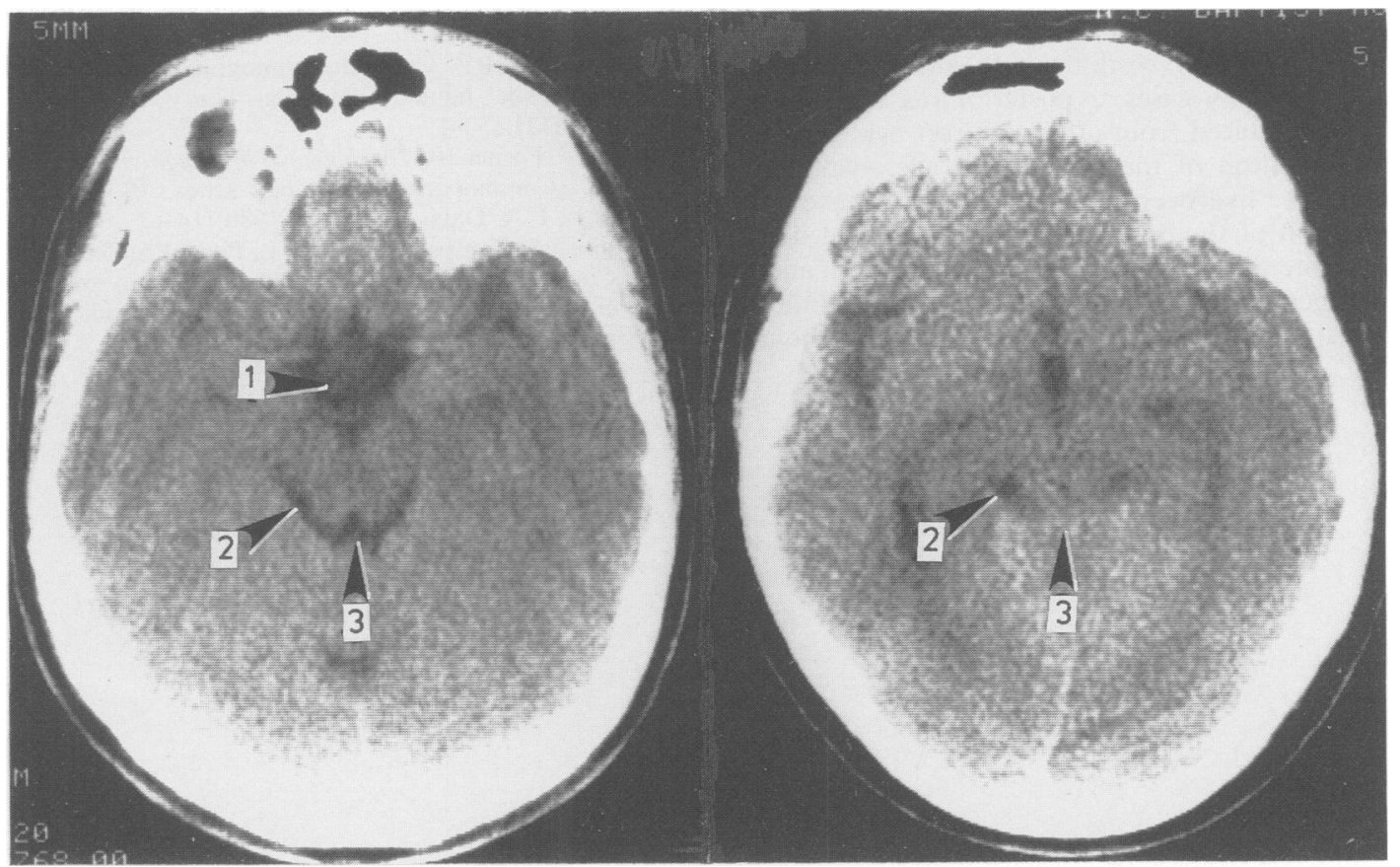

Fig 2 (left) Normal uninfused CT scan demonstrating the basilar cisterns $(1=$ suprasellar cistern, $2=$ ambient cistern, 3 = quadrigeminal plate cistern), and (right) an example of upward transtentorial herniation with obliteration of the quadrigeminal plate cistern (3) and sparing of the ambient cistern (2). 
infratentorial pressure are identified by loss of the suprachiasmatic and circummesencephalic cisterns (basilar cisterns) (fig 2, right). The ventricular system may be symmetrically small as in diffuse cerebral oedema, or the lateral ventricles including the temporal horns may be enlarged as in obstructive (noncommunicating) hydrocephalus. Decompression of the spinal and posterior fossa compartments from lumbar puncture may cause the medial temporal lobes to impact into the tentorial hiatus bilaterally (bilateral uncal herniation).

We believe the strongest contraindication to lumbar puncture is a posterior fossa mass. Any increase in pressure in the posterior fossa may move the cerebellar tonsils into the region of the foramen magnum, predisposing the patient to tonsillar herniation whenever even a small amount of cerebrospinal fluid is removed from the spinal compartment. Unfortunately, the posterior fossa and foramen magnum are difficult areas to image by computed tomography, and direct demonstration of the posterior fossa mass may be compromised by bone and motion artifact, isodensity, volume averaging from thick slices, or failure to infuse the patient. An asymmetrical lesion such as a cerebellar hemispherical mass may shift the fourth ventricle laterally; anterior and posterior compartment masses may compress the fourth ventricle and shift it anteriorly or posteriorly. Shifts of the fourth ventricle produced by low and high posterior fossa masses may be difficult to image in the axial plane. In many cases, a posterior fossa mass effect must be deduced from other, indirect evidence, such as obliteration of the fourth ventricle, symmetrical obstructive hydrocephalus, or upward transtentorial herniation of the cerebellar vermis. Upward herniation obliterates the superior cerebellar cistern and the quadrigeminal plate cistern caudal to the midbrain while sparing the ambient cisterns lateral to the mid- brain (fig 2, left). Upward herniation may be a very subtle finding on the CT scan, and a high index of suspicion is required.

In summary, we have presented a case of cerebral herniation following lumbar puncture, and have suggested criteria that may help guide the clinician assessing data obtained from an "abnormal" CT scan to define more accurately the risk-to-benefit ratio for lumbar puncture.

The authors thank Judith MacMillan for editorial assistance, as well as Alma Doub and Libby Childress for manuscript preparation.

\section{References}

1 Wood JH. Cerebrospinal fluid: techniques of access and analytical interpretation. In: Wilkins RH, Rengachary SS, eds. Neurosurgery Vol 1. New York, McGraw-Hill Book Company, 1985;161-74.

2 Cushing H. Some aspects of the pathological physiology of intracranial tumors. Boston Med Surg J 1909;161:71-80.

3 Korein J, Cravioto H, Leicach M. Revaluation of lumbar puncture. A study of 129 patients with papilloedema or intracranial hypertension. Neurology 1959;9:290-7.

4 Lafforgue JL, de Coninck L, Cales J, Bagot M, Sabathié M. A propos de la ponction lombaire: indications et limites. Cah Anesth. 1984;32:113-7.

5 Humphreys RP. Computed tomography and the early diagnostic lumbar puncture. Can Med Assoc $J$ 1979;121:150-1.

6 Plum F, Posner JB. The diagnosis of stupor and coma, 3rd ed. (Contemporary neurology series, 19.) Philadelphia, F.A. Davis Company, 1980;114-6.

7 Sadhu VK, Sampson J, Haar FL, Pinto RS, Handel SF. Correlation between computed tomography and intracranial pressure monitoring in acute head trauma patients. Radiology 1979;133:507-9. 